\title{
Theoretical Models of Dark Energy
}

\author{
Varun Sahni \\ Inter-University Centre for Astronomy \& Astrophysics, \\ Puné 411 007, India
}

\begin{abstract}
Observations of high redshift type Ia supernovae indicate that the universe is accelerating, fueled by an unknown form of 'dark energy' having large negative pressure $p<0$. The simplest example of dark energy is the cosmological constant $(p=-\rho \equiv-\Lambda / 8 \pi G)$. The cosmological constant arises at a fundamental level from one-loop quantum effects which generate a $\Lambda$-term many orders of magnitude larger than the observed value of dark energy $\sim 10^{-47} \mathrm{GeV}^{4}$. This leads to the 'cosmological constant problem'. Dynamical models of dark energy include scalar fields with exponential and power law potentials. Dark energy can also be generated in extra-dimensional 'braneworld' models. Model-independent methods which attempt to reconstruct dark energy from supernova observations are discussed.
\end{abstract}

\section{The observational case for dark energy}

Observations of angular anisotropies in the cosmic microwave background on degree scales indicate that the universe is close to being spatially flat with (Wang, Tegmark \& Zaldarriaga 2001) $\Omega_{\text {total }}=1.0_{-0.05}^{+0.06}$. This value for the total mass density in the universe is considerably larger than the value for the density in clustered matter $\Omega_{m} \simeq 0.15-0.3$ (inferred from measurements of rotation curves for individual galaxies as well as dynamical and SunyaevZeldovich measurements of masses in galaxy clusters). The discrepancy between $\Omega_{m} \simeq 1 / 3$ and $\Omega_{\text {total }}=1$ can be accounted for if roughly $2 / 3$ of the matter in the universe resides in a smooth (unclustered) component. This result is supported by recent observations of high redshift supernovae which indicate that the universe is accelerating, fueled by a cosmological constant or some other form of dark energy which has a negative pressure $p<0$ (see article by B. Schmidt in this volume). It should be emphasized that, despite 
considerable evidence for dark matter and mounting observational evidence for dark energy, the nature of both dark matter and dark energy is a mystery at present. This mystery deepens when we note that current estimates of the cosmic baryon density (based on arguments related to the cosmological nucleosynthesis of light elements and recent HST-based measurements of the Hubble parameter) yield the estimate (Albrecht, Frieman and Trodden 2001) $\Omega_{b} \simeq 0.04$ which strongly suggests that both the dark matter and the dark energy must be non-baryonic in origin.

The supernova observations are linked to the principle that the light flux received from a distant object is related to its distance from us $d_{L}$ and to its absolute luminosity $\mathcal{L}$ through the relation

$$
F=\frac{\mathcal{L}}{4 \pi d_{L}^{2}} .
$$

Within the Newtonian framework the geometry of space is Euclidean and the distance to an object is simply $d_{L}=\sqrt{x^{2}+y^{2}+z^{2}}$. In general relativity on the other hand the geometry of space is generally non-Euclidean, the luminosity distance to an object located at redshift $z$ will therefore depend both upon the geometry of space as well as the expansion history of the universe. Since the expansion of the universe depends upon the kind of matter it contains, the distance to a supernova at redshift $z$, can vary greatly from one cosmological model to another. In the absence of systematic effects which could diminish the light flux from distant objects, the dimming of light from high redshift supernovae is taken to imply that the universe is accelerating, the acceleration being caused by a matter source having the unusual property that its pressure is significantly negative. To appreciate this, consider again the situation within the Newtonian perspective wherein the force experienced by a point mass placed on the surface of a solid sphere of radius ' $a$ ' and uniform density $\rho$ and pressure $p$ is $F=-G m M / a^{2}$, where $M=4 \pi G a^{3} \rho / 3$. Substituting $F=m \ddot{a}$ we obtain

$$
\ddot{a}=-\frac{4 \pi G}{3} \rho a,
$$

which demonstrates that deceleration is the normal fate of a test particle in Newtonian cosmology. In general relativity (2) is replaced by

$$
\ddot{a}=-\frac{4 \pi G}{3} a \sum_{i}\left(\rho_{i}+3 p_{i}\right),
$$


where the summation is over all forms of matter present in the universe. The change $(2) \rightarrow(3)$ is caused by the important fact that in general relativity, the gravitational force law is determined not by the density of matter alone, but by the matter energy-momentum tensor $T_{i k}$ through the Einstein field equations $G_{i k}=8 \pi G T_{i k}$, which reduce to (3) for the homogeneous and isotropic Friedmann-Robertson-Walker (FRW) space-time describing our universe.

Eqn. (3) together with its companion equation

$$
H^{2} \equiv\left(\frac{\dot{a}}{a}\right)^{2}=\frac{8 \pi G}{3} \sum_{i} \rho_{i}-k / a^{2}
$$

completely describes the dynamics of the universe $\left(k / a^{2}\right.$ is the Gaussian curvature of space). Assuming for simplicity that the universe is dominated by a single matter component having equation of state $p=w \rho,(3)$ reduces to

$$
\ddot{a}=-\frac{4 \pi G}{3}(1+3 w) \rho
$$

which establishes that the universe decelerates if $w>-1 / 3$ and accelerates if $w<-1 / 3$. (For $w=-1 / 3$ the universe coasts at the steady rate $a \propto t$.) Matter with $w<-1 / 3$ is called 'dark energy' to differentiate it from 'dark matter' which makes up $\sim 1 / 3$ of the total density of the universe and for which $w \geq 0$. The simplest form of dark energy is the cosmological constant ' $\Lambda$ ' for which $w=-1$ and the energy-momentum tensor has the covariant form $T_{i}^{k}=\Lambda \delta_{i}^{k}$, where $\Lambda / 8 \pi G=\rho_{\Lambda}$ is the energy density associated with $\Lambda$.

In the case of a two-component universe consisting of pressure-less matter $(w=0)$ and a cosmological constant, (3) can be rewritten in the form of a force law:

$$
\mathcal{F}=-\frac{G M}{R^{2}}+\frac{\Lambda}{3} R, \quad(R \equiv a)
$$

which demonstrates that the cosmological constant gives rise to a repulsive force whose value increases with distance, and which could therefore be responsible for the current acceleration of the universe.

Eqn. (4) can be used to develop an expression for the Hubble parameter $H \equiv \dot{a} / a$ in terms of the cosmological redshift $z=a_{0} / a(t)-1$ :

$$
H(z)=H_{0}\left[\Omega_{m}(1+z)^{3}+\Omega_{X}(1+z)^{3(1+w)}\right]^{1 / 2},
$$

where $H_{0}=H(z=0)$ is the present value of the Hubble parameter. $\Omega_{m}=$ $8 \pi G \rho_{0 m} / 3 H_{0}^{2}, \Omega_{X}=8 \pi G \rho_{0 X} / 3 H_{0}^{2}$, describe the dimensionless density of 
matter and dark energy respectively, $\Omega_{m}+\Omega_{X}=1$ is assumed. For the cosmological constant $w=-1$ and $\Omega_{\Lambda}=\Lambda / 3 H_{0}^{2}$. (We neglect the presence of radiation which does not play a significant role at $z \lesssim$ few.) The luminosity distance in a spatial flat homogeneous and isotropic universe is described by the simple expression

$$
d_{L}(z)=(1+z) \int_{0}^{z} \frac{d z^{\prime}}{H\left(z^{\prime}\right)}
$$

with $H(z)$ given by (7). The luminosity distance is shown in Fig. 1 for a number of cosmological models with varying amounts of $\Omega_{m} \& \Omega_{\Lambda}$. The limiting case $\Omega_{m}=1, \Omega_{\Lambda}=0$ corresponds to a matter dominated universe, (also called the standard cold dark matter model - SCDM) in which the universe decelerates as a weak power law

$$
a(t) \propto t^{2 / 3} .
$$

The other extreme example $\Omega_{\Lambda}=1, \Omega_{m}=0$ describes the de Sitter universe (also known as steady state cosmology) which accelerates at the steady rate

$$
a(t) \propto \exp \sqrt{\frac{\Lambda}{3}} t
$$

From Fig. $\quad 1$ we see that a galaxy at redshift $z=3$ will appear 9 times brighter in a flat matter dominated universe than it will in de Sitter space!

Fig. 1 Caption: The luminosity distance $d_{L}$ (in units of $H_{0}^{-1}$ ) is shown as a function of cosmological redshift $z$ for spatially flat cosmological models with a cosmological constant $\Omega_{m}+\Omega_{\Lambda}=1$. Heavier lines correspond to larger values of $\Omega_{m}$. The dashed line shows the luminosity distance in the spatially flat de Sitter universe $\left(\Omega_{\Lambda}=1\right)$. (Reproduced from Sahni and Starobinsky 2000.)

\section{Cosmic coincidence and dark energy}

Observations of several dozen high redshift type Ia supernovae indicate that the matter content of the universe is best described by (Perlmutter et al. 1999), Riess et al. 1998) $\Omega_{\Lambda} \simeq 2 / 3, \Omega_{m} \simeq 1 / 3$. The expansion of such a 
universe lies in between the two extremes (9) \& (10) and is described by (Sahni and Starobinsky 2000)

$$
a(t) \propto\left(\sinh \frac{3}{2} \sqrt{\frac{\Lambda}{3}} c t\right)^{2 / 3} .
$$

It is easy to see that (11) interpolates between $a \propto t^{2 / 3}$ in the past and $a \propto \exp \sqrt{\Lambda / 3} t$ in the future. If dark energy is described by the constant equation of state $w=p_{X} / \rho_{X}$, then the instant the universe stops decelerating and begins to accelerate $(\ddot{a}=0)$ is described by the redshift $z_{\mathrm{a}}$, where

$$
\left(1+z_{\mathrm{a}}\right)^{-3 w}=-(1+3 w) \frac{\Omega_{X}}{\Omega_{m}} .
$$

Another important redshift describes the epoch when $\rho_{X}\left(z_{\text {eq }}\right)=\rho_{m}\left(z_{\text {eq }}\right)$

$$
\left(1+z_{\mathrm{eq}}\right)^{3}=\left(\frac{\Omega_{X}}{\Omega_{m}}\right)^{-1 / w}
$$

From (12) \& (13) we find that the acceleration of the universe is a fairly recent phenomenon since, for the cosmological constant $\left(\Omega_{\Lambda}=0.7, \Omega_{m}=0.3\right) z_{\mathrm{a}} \simeq$ $0.73, z_{\mathrm{eq}} \simeq 0.37$ so that $z_{\mathrm{a}}>z_{\mathrm{eq}}$. This illustrates the 'cosmic coincidence' puzzle according to which we appear to live during a special epoch when the densities in dark energy and in matter are almost equal. Models of dark energy in which $w=$ constant (which includes $\Lambda$ ) also suffer from the 'fine tuning problem'. The density of matter in an expanding universe decreases according to the general rule

$$
\rho \propto a^{-3(1+w)}
$$

(Eqn. (14) can be derived from the conservation equation $T_{i}{ }^{k}{ }_{k}=0$ which, in turn, can be recast as $d E=-p d V$ where $E=\rho \times V, V(t) \propto a^{3}(t)$.)

From (14) we find that $\rho_{\Lambda}=$ constant if $w=-1$, while $\rho_{m}(t) \propto a^{-3}$ if $w=0$ (pressure-less matter), and $\rho_{r}(t) \propto a^{-4}$ if $w=1 / 3$ (radiation). Since the energy density associated with the cosmological constant does not change with time while the density of both matter and radiation declines rapidly as the universe expands, it follows that both the matter and radiation densities must have been significantly greater than $\rho_{\Lambda}$ in the past. Since 
$\rho_{\Lambda}=\Lambda / 8 \pi G \simeq 10^{-47} \mathrm{GeV}^{4}$, one finds that $\rho_{\Lambda} / \rho_{r} \simeq 10^{-123}$ at the Planck time (when the temperature of the universe was $T \sim 10^{19} \mathrm{GeV}$ ), while $\rho_{\Lambda} / \rho_{r} \simeq$ $10^{-55}$ at the time of the electroweak phase transition $(T \sim 100 \mathrm{GeV})$. Thus the value of the cosmological constant should have been set to an accuracy of better than one part in $10^{123}$ at the start of the big bang $\left(z=a_{0} / a-1 \sim 10^{32}\right)$ in order for the universe to begin accelerating at $z \sim 1$ !

\section{$3 \quad$ Vacuum energy and $\Lambda$}

The fine tuning problem is severely aggravated by the fact that within the framework of quantum field theory, the value of the cosmological constant is very large (formally infinite). This has to do with the observation that vacuum fluctuations generate a cosmological constant whose value (for massive scalar fields) is

$$
\frac{\Lambda_{\mathrm{vac}}}{8 \pi G} \equiv\left\langle T_{00}\right\rangle_{\mathrm{vac}} \propto \int_{0}^{\infty} \sqrt{k^{2}+m^{2}} k^{2} d k .
$$

The integral in (15) diverges as $k^{4}$ which leads to an infinite value for the oneloop 'vacuum energy density ' associated with scalar fields. (Similar contributions also arise from other fundamental fields existing in nature.) Assuming that quantum gravity will provide a fundamental cutoff to all physical processes on the scale $k \sim k_{\mathrm{Pl}} \sim 10^{19} \mathrm{GeV}$, we get a finite, but very large value for the vacuum energy: $\left\langle T_{00}\right\rangle_{\mathrm{vac}} \simeq c^{5} / G^{2} \hbar \sim 10^{76} \mathrm{GeV}^{4}$ which is 123 orders of magnitude larger than the currently observed value $\rho_{\Lambda} \simeq 10^{-47} \mathrm{GeV}^{4}$.

In super-symmetric models bosons and fermions (of identical mass) contribute equally but with opposite sign to the vacuum expectation value of physical quantities. It might therefore be hoped that $\Lambda_{\mathrm{vac}} \simeq 0$ within the super-symmetric framework. However super-symmetry is broken at the low temperatures prevailing in the universe today and is restored at $T=T_{\mathrm{SUSY}}>$ $1 \mathrm{TeV}$. One therefore expects the cosmological constant to vanish in the early universe when $T \gg 1 \mathrm{TeV}$, but to reappear later when the temperature drops below $T_{\mathrm{SUSY}}$. This is exactly the opposite of what one desires of nature, since a large cosmological $\Lambda$-term at an early epoch could drive inflation while a small current value of $\Lambda$ could give rise to dark energy.

An interesting method for generating a small vacuum energy was suggested by Zeldovich, who remarked that after the removal of one-loop quan- 
tum divergences, one would still be left with finite contributions to the vacuum energy at the two loop level. At this level the gravitational interactions between particle \& antiparticle would lead to a vacuum energy density (Zeldovich, 1968)

$$
\rho_{\Lambda} \sim \frac{G m^{2}}{\hbar c} m\left(\frac{m c}{\hbar}\right)^{3} .
$$

(One way of understanding this result is by noting that the interaction energy density is typically $\epsilon_{v a c} \equiv \rho_{v a c} c^{2} \sim \frac{G m^{2}}{\lambda} / \lambda^{3}$ where $\lambda=\hbar / m c$ is the mean separation between particle and antiparticle.) Although the proton-antiproton (electron-positron) contribution gives a very large (small) value for $\rho_{\Lambda}$, the pion-antipion mass gives just the right value $\rho_{\Lambda}=\frac{1}{(2 \pi)^{4}} \rho_{P}\left(m_{\pi} / M_{P}\right)^{6} \simeq$ $1.3 \times 10^{-123} \rho_{P}=6.91 \times 10^{-30} \mathrm{~g} \mathrm{~cm}^{-3} \cdot\left(\rho_{P} \sim c^{5} / G^{2} \hbar \sim 10^{76} \mathrm{GeV}^{4}\right.$ is the Planck density.) Other speculative ideas for generating a small value for the dark energy include the following (Sahni and Starobinsky 2000):

- It is possible to generate a sufficiently small value of $\Lambda$ through a suitable combination of the fundamental constants. Combining the fine structure constant $\alpha$ with the Planck density $\rho_{P}$ we can get $\rho_{\Lambda}=$ $\frac{\rho_{P}}{\left(2 \pi^{2}\right)^{3}} e^{-2 / \alpha} \simeq 1.2 \times 10^{-123} \rho_{P}=6.29 \times 10^{-30} \mathrm{~g} \mathrm{~cm}^{-3}$.

- It is interesting that the mass scale associated with the scale of supersymmetry breaking in some models, $M_{\mathrm{SUSY}} \sim 1 \mathrm{TeV}$, lies midway between the Planck scale and $10^{-3} \mathrm{eV}$. The small observed value of the cosmological constant $\rho_{\Lambda} \simeq\left(10^{-3} \mathrm{eV}\right)^{4}$ might therefore be associated with the vacuum in a theory which had a fundamental mass scale $M_{X} \simeq M_{\mathrm{SUSY}}^{2} / M_{\mathrm{Pl}}$, so that $\rho_{\mathrm{vac}} \sim M_{X}^{4} \sim\left(10^{-3} \mathrm{eV}\right)^{4}$.

\subsection{Spontaneous symmetry breaking and $\Lambda$}

A large cosmological constant can also arise in field theory models with spontaneous symmetry breaking. As an example consider the symmetry breaking Lagrangian

$$
\begin{aligned}
\mathcal{L} & =\frac{1}{2} g^{i j} \partial_{i} \phi \partial_{j} \phi-V(\phi), \\
V(\phi) & =V_{0}-\frac{1}{2} \mu^{2} \phi^{2}+\frac{1}{4} \lambda \phi^{4} .
\end{aligned}
$$


The state $\phi=0$ is symmetric but unstable, as a result the system will tend to settle into the stable ground state $\phi=+\sigma$ or $\phi=-\sigma$, where $\sigma=\sqrt{\mu^{2} / \lambda}$ - a state which breaks the reflection symmetry present in the Lagrangian (17). Setting $V_{0}=0$ results in a large negative cosmological constant $\Lambda_{\text {eff }}=$ $V(\phi=\sigma)=-\mu^{4} / 4 \lambda$ associated with the broken symmetry state. A large negative cosmological constant spells disaster for cosmology since a universe with $\Lambda \ll 0$ will re-collapse almost immediately after the initial big bang. To remedy this situation one 'regularizes' the vacuum state to $V_{0} \sim \mu^{4} / 4 \lambda$ which leads to a more moderate value $\Lambda_{\text {eff }} / 8 \pi G=V_{0}-\mu^{4} / 4 \lambda \simeq 10^{-47} \mathrm{GeV}^{4}$. Clearly this involves a considerable degree of fine tuning. One must therefore conclude that even within the framework of classical field theory, there is no real understanding of why the observed value of the cosmological constant is so much smaller than the scale of one of the vacuum condensates which could occur in nature such as $M_{\mathrm{GUT}}^{4} \sim\left(10^{15} \mathrm{GeV}\right)^{4}, M_{\mathrm{W}}^{4} \sin ^{4} \theta_{\mathrm{W}} /(4 \pi \alpha)^{2} \sim$ $(175 \mathrm{GeV})^{4}$, or $f_{\pi}^{4} \sim(100 \mathrm{MeV})^{4}$ (Weinberg 1989, Albrecht, Frieman and Trodden 2001).

Fig. 2 Caption: Spontaneous symmetry breaking in many field theory models takes the form of the 'Mexican top hat' potential shown above. The dashed line shows the potential before the cosmological constant has been 'renormalized' and the solid line after. (Reproduced from Sahni and Starobinsky 2000.)

\section{Dynamical Models of Dark Energy}

In the previous section we showed that quantum effects and effects associated with symmetry breaking usually give rise to a very large value of the vacuum energy. This result can be reconciled with the extremely small value of the dark energy observed today only if there exists some (presently undiscovered) symmetry which sets the value of $\Lambda$ to exactly zero, or else due to some (currently unknown) cancellation mechanism which operates in the universe.

Theoretical models of dark energy usually make either of these two assumptions and set $\Lambda_{\mathrm{vac}}=0$ before tackling the fundamental issues relating to the 'fine tuning' and 'cosmic coincidence' problems discussed earlier in this paper.

To resolve the fine-tuning problem, dynamical models of dark energy usually do away with the assumptions $\rho, w=$ constant, and allow the density 
of dark energy and its equation of state to be functions of the cosmic time. The reason for this is that models with $w=$ constant generically suffer from the fine tuning problem since, for the observational upper-bound $w \leq-0.8$ (Saini, Raychaudhury, Sahni and Starobinsky, 2000), the ratio of the density in dark energy to the density in matter $\rho_{X} / \rho_{m}=\left(\Omega_{0 X} / \Omega_{0 m}\right) \times(1+z)^{3 w}$ decreases rapidly with redshift and becomes a very small quantity at early times.

A dynamical model of dark energy therefore belongs to one of the following three general classes (Sahni and Starobinsky 2000):

(1) Kinematic models.

Dark energy is assumed to be a function of either the cosmic time $t$ or the scale factor $a(t)$ of the FRW cosmological model.

(2) Hydrodynamic models.

Dark energy is described by a barotropic fluid with some equation of state $w=p / \rho$ (dissipative terms may also be present).

It is easy to show that the simplest kinematic models

$$
\Lambda \equiv 8 \pi G \rho_{\Lambda}=f(a)
$$

are equivalent to hydrodynamic models based on an ideal fluid with an equation of state

$$
w=p / \rho=-\left(1+\frac{1}{3} \frac{d \ln \rho_{\Lambda}}{d \ln a}\right) .
$$

(3) Field-theoretic models. Dark energy is assumed to be a new physical field described by a phenomenological Lagrangian.

We shall concentrate on field-theoretic models of dark energy in the rest of this paper since we consider them to be the most physically compelling.

\subsection{Field theoretic models of dark energy}

An interesting example of dark energy which need not run into the fine tuning problem is provided by scalar fields which couple minimally to gravity and whose energy momentum tensor is

$$
\rho \equiv T_{0}^{0}=\frac{1}{2} \dot{\phi}^{2}+V(\phi), P \equiv-T_{\alpha}^{\alpha}=\frac{1}{2} \dot{\phi}^{2}-V(\phi) .
$$

A scalar field rolling down its potential slowly, generates a time-dependent $\Lambda$-term since $P \simeq-\rho \simeq-V(\phi)$ if $\dot{\phi}^{2} \ll V(\phi)$. Potentials satisfying $\Gamma \equiv$ 
$V^{\prime \prime} V /\left(V^{\prime}\right)^{2} \geq 1$ possess the important property that scalar fields moving under their influence approach a common evolutionary path from a wide range of initial conditions (Ratra and Peebles 1988, Wetterich 1988, Ferreira and Joyce 1997, Zlatev, Wang and Steinhardt 1999). In these so-called 'tracker' models, the scalar field density 'tracks' the dominant matter density driving the expansion of the universe. A good example is provided by the exponential potential $V(\phi)=V_{0} \exp \left(-\lambda \phi / M_{P}\right), M_{P}=1 / \sqrt{8 \pi G}$ (Ratra and Peebles 1988, Wetterich 1988, Ferreira and Joyce 1997) for which

$$
\frac{\rho_{\phi}}{\rho_{B}+\rho_{\phi}}=\frac{3\left(1+w_{B}\right)}{\lambda^{2}}=\text { constant }<0.2,
$$

where $\rho_{B}$ is the background matter density and $w_{B}$ is its equation of state. The lower bound $\rho_{\phi} / \rho_{\text {total }}<0.2$ is associated with nucleosynthesis constraints which prevent the dark energy density from being large at the time when the light elements in the universe were being synthesized $(t \sim f e w$ sec.). Since the ratio $\rho_{\phi} / \rho_{\text {total }}$ remains unchanged as the universe evolves, exponential potentials are unable to generate a large amount of dark energy at the present epoch. However a simple generalization of the exponential potential achieves this. The class of dark energy potentials (Sahni and Wang 2000)

$$
V(\phi)=V_{0}[\cosh \lambda \phi-1]^{p},
$$

satisfies $w_{\phi} \simeq w_{B}$ at early times when the form of the potential approaches an exponential. The change in the form of the potential from an exponential to a power law at late times ensures that the scalar field oscillates rapidly and that the effective equation of state of dark energy changes to $\left\langle w_{\phi}\right\rangle=(p-1) /(p+1)$ at present. Consequently (22) can describe 'tracking' dark energy (if $p \leq 1 / 2$ ) and 'tracking' 'cold' dark matter (CDM) (if $p=1$ ) as demonstrated in Fig. 3. (For other applications of exponential potentials see Amendola 2000, Bareiro, Copeland and Nunes 2000.)

Fig. 3 Caption: The evolution of the dimensionless density parameter for the CDM field $\Omega_{\psi}$ (dashed line) and dark energy $\Omega_{\phi}$ (thin solid line) is shown as a function of the cosmic redshift. The potential responsible for both dark energy and CDM is (22) with $p=0.2$ describing dark energy and $p=1$ describing CDM. Baryon (dash-dotted line) and radiation densities (thick solid line) are also shown. The fine tuning problem is largely ameliorated in this model since the initial value of the dark energy is of the same order as the radiation density. 
A different example of tracker dark energy is provided by the potential (Ratra and Peebles, 1988) $V(\phi)=V_{0} / \phi^{\alpha}$. In this case the ratio of the energy density of the scalar field to that of radiation/matter gradually increases during the radiation and matter dominated epochs $\rho_{\phi} / \rho_{B} \propto t^{4 /(2+\alpha)}$, since its equation of state remains somewhat smaller than the background value $w_{\phi}=\left(\alpha w_{B}-2\right) /(\alpha+2)$. This process allows the scalar field to eventually dominate the density of the universe giving rise to the current epoch of accelerated expansion. (Supernova observations lead to the strong constrain $\alpha \leq 2$.)

Some scalar field potentials which can give rise to the properties of dark energy have been listed in Table 1 (Sahni 2002). Several of them have been inspired by field theoretic ideas including supersymmetric gauge theories and supergravity, pseudo-goldstone boson models, etc. One should point out that accelerated expansion can also arise in models with: (i) topological defects including a frustrated network of cosmic strings $(w \simeq-1 / 3)$ and domain walls $(w \simeq-2 / 3)$ (Bucher and Spergel 1998); (ii) scalar field Lagrangian's with non-linear kinetic terms and no potential term (k-essence) (AramdarizPicon, Mukhanov and Steinhardt, 2000); (iii) vacuum polarization associated with an ultra-light non-minimally coupled scalar (Sahni and Habib, 1998); (iv) brane-world models (Copeland, Liddle and Lidsey 2000, Huey and Lidsey 2001, Sahni, Sami and Souradeep 2002, Deffayet, Dvali and Gabadadze 2001, Sahni and Shtanov 2002).

\section{$5 \quad$ Reconstructing dark energy}

As we showed in the previous section, the fine tuning problem faced by the cosmological constant can be significantly resolved if dark energy depends upon time. However, given the large number of dark energy models and the current absence of a fundamental theory of high energy physics carrying a unique prescription for dark energy, it becomes meaningful to look for model independent methods which could be used to reconstruct the properties of dark energy directly from observations. 


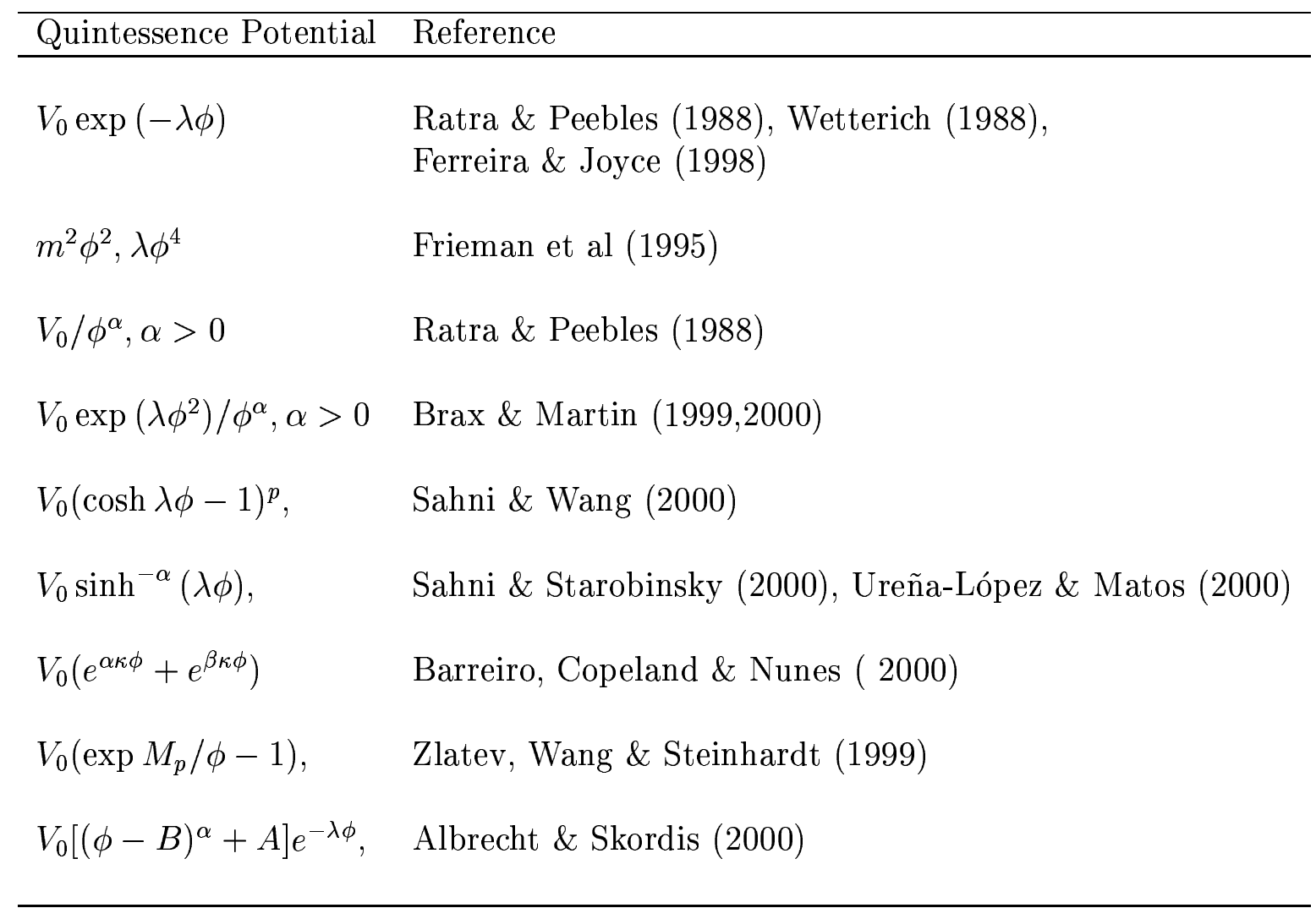

Table 1:

\subsection{Equation of state reconstruction}

As shown by Saini, Raychaudhury, Sahni and Starobinsky (2000), one can reconstruct the dark energy potential $V(\phi)$ and its equation of state $w=p / \rho$ if we know the form of the luminosity distance $d_{L}(z)$. This reconstruction exercise is feasable because the scalar field potential as well as its equation of state are functions of the Hubble parameter and its derivative

$$
\frac{8 \pi G}{3 H_{0}^{2}} V(x)=\frac{H^{2}}{H_{0}^{2}}-\frac{x}{6 H_{0}^{2}} \frac{d H^{2}}{d x}-\frac{1}{2} \Omega_{m} x^{3}
$$




$$
\begin{aligned}
\frac{8 \pi G}{3 H_{0}^{2}}\left(\frac{d \phi}{d x}\right)^{2} & =\frac{2}{3 H_{0}^{2} x} \frac{d \ln H}{d x}-\frac{\Omega_{m} x}{H^{2}}, x=1+z \\
w_{\phi}(x) \equiv \frac{p}{\varepsilon} & =\frac{(2 x / 3) d \ln H / d x-1}{1-\left(H_{0}^{2} / H^{2}\right) \Omega_{m} x^{3}}
\end{aligned}
$$

The Hubble parameter, in turn, is related to the luminosity distance through (8) which can be rewritten as

$$
H(z)=\left[\frac{d}{d z}\left(\frac{d_{L}(z)}{1+z}\right)\right]^{-1} .
$$

It is therefore possible to reconstruct both the dark energy potential $V(\phi)$ as well as the equation of state $w(z)$, if the luminosity distance $d_{L}(z)$ is known from observations. This method when applied to the luminosity distance $d_{L}(z)$ determined from high redshift supernovae gives the results shown in Fig. 4. We see that the current value of the equation of state of dark energy is constrained by the upper-bound $w_{0} \leq-0.8$ at the $90 \%$ confidence level if the value of $\Omega_{m}$ is known exactly.

Fig. 4 Caption: The equation of state of dark energy is reconstructed from observations of Type Ia high redshift supernovae in a model independent manner. The equation of state satisfies $-1 \leq w_{\phi} \leq-0.8$ at $z=0$; and $-1 \leq w_{\phi} \leq-0.46$ at $z=$ $0.83(90 \% \mathrm{CL}), \Omega_{m}=0.3$ is assumed. The best fit dark energy is shown by the solid line. A cosmological constant $(w=-1)$ is seen to be consistent with the observations. (Reproduced from Saini, Raychaudhury, Sahni and Starobinsky 2000.)

\subsection{The Statefinder}

A complementary model-independent diagnostic of dark energy called 'Statefinder' has recently been suggested by Sahni, Saini, Starobinsky and Alam (2002). The Statefinder consists of the parameter pair $\{r, s\}$ where the parameter $r=\dddot{a} / a H^{3}$ forms the next logical step in the hierarchy of geometrical cosmological parameters used to study the Universe after $H=\dot{a} / a$ and $q=-\ddot{a} / a H^{2}$. The parameter $s(z)$ is a linear combination of $q$ and $r$ chosen in such a way that it does not depend upon the dark energy density $\Omega_{X}(z)$ :

$$
s=\frac{r-1}{3(q-1 / 2)}=1+w-\frac{1}{3} \frac{\dot{w}}{w H} .
$$


The Statefinder pair $\{r, s\}$ has the remarkable property that the value of $r$ stays pegged at $r=1$ throughout the entire matter dominated epoch and at all future times in a cosmological constant driven universe (LCDM) irrespective of the current value of $\Omega_{m}$. The value of $s$ stays pegged to $s=0$. This property helps distinguish the cosmological constant from time-dependent sources of dark energy for which $r, s$ are usually functions of time.

Fig. 5 shows the results of an analysis which uses projected supernova observations from the proposed SuperNovae Acceleration Probe (SNAP) which is expected to observe approximately 2000 Type Ia supernovae within a year up to a redshift $z \sim 2$, (see table II). Our results are obtained from 1000 realizations of a SNAP-type experiment for the 'mean Statefinder statistic' defined as

$$
\begin{aligned}
\bar{r} & =\frac{1}{z_{\max }} \int_{0}^{z_{\max }} r(z) d z, \\
\bar{s} & =\frac{1}{z_{\max }} \int_{0}^{z_{\max }} s(z) d z
\end{aligned}
$$

where $z_{\max }=1.7$. The statistical uncertainty in the magnitude of $\mathrm{SNe}$ is assumed to be a constant over redshift and is given by $\sigma_{\mathrm{mag}}=0.15$. The results of Fig. 5 clearly show that the Statefinder diagnostic is very sensitive to different models of dark energy and can reliably be used to discriminate between a cosmological constant and several time-dependent forms of dark energy.

Table 2: SNAP specifications for one year period of observations

\begin{tabular}{ccccc} 
Redshift Interval & $z=0-0.2$ & $z=0.2-1.2$ & $z=1.2-1.4$ & $z=1.4-1.7$ \\
\hline Number of SNe & 50 & 1800 & 50 & 15
\end{tabular}

Fig. 5 Caption: Confidence levels at $1 \sigma, 2 \sigma, 3 \sigma$ of $\bar{r}$ and $\bar{s}$ computed from 1000 SNAP-type experiments probing a LCDM fiducial model with $\Omega_{0 m}=0.3, \Omega_{0 \Lambda}=0.7$. The filled circles represent the values of $\bar{r}$ and $\bar{s}$ for the dark energy potential $V(\phi) \propto \phi^{-\alpha}$ with $\alpha=1,2,3,4,5,6$ (bottom to top). The filled triangles represent dark energy with a constant equation of state $w=-2 / 3,-1 / 2,-1 / 3,0$ (bottom to top). It is interesting that 
all inverse power-law models lie well outside of the three sigma contour centered around the LCDM model. (Reproduced from Sahni, Saini, Starobinsky and Alam 2002.)

\section{Conclusions}

In this article I have attempted to briefly review some of the issues relating to a new form of gravitating 'dark energy' which might exist in the universe. The properties of dark energy are intimately related to its equation of state $w=p / \rho$. Models of dark energy in which $w$ is a constant invariably run into a 'fine tuning problem' since the initial value of the dark energy must be 'tuned' to be a tiny fraction of the total matter density in the universe at early times. Dark energy models built around 'tracker potentials' circumvent this problem. Dark energy can also arise as the vacuum polarization of fundamental fields, in topological defect models and in higher dimensional braneworld cosmology. The properties of dark energy are currently derived mainly from observations of high redshift supernovae (Sn) and the cosmic microwave background (CMB). In the future, high quality astrophysical observations of: (i) weak and strong gravitational lensing, (ii) the Lyman- $\alpha$ forest, (iii) gravitational clustering of galaxies, clusters and the large scale structure of the universe, will complement Sn and CMB studies and help provide valuable constraints on theoretical models of dark energy.

\section{References}

Albrecht, A., Frieman, J.A. and Trodden, M. 2001, astro-ph/0111080.

Albrecht, A. and Skordis, C. 2000, Phys. Rev. Lett. 84, 2076.

Amendola, L. 2000, Phys. Rev. D 62043511.

Aramdariz-Picon, C., Mukhanov, V., Steinhardt, P.J., 2000, Phys. Rev. Lett. 85, 4438. Barreiro, T., Copeland, E.J. and Nunes, N.J. 2000, Phys. Rev. D 61, 127301.

Brax, P. and Martin, J. 2000, Phys. Rev. D 61, 103502.

Brax, P. and Martin, J. 1999, Phys. Lett. B 468, 40.

Bucher, M. and Spergel, D. 1999, Phys. Rev. D 60, 043505.

Copeland, E.J., Liddle, A.R. and Lidsey, J.E. 2001, Phys. Rev. D 64023509.

Deffayet, C., Dvali, G. and Gabadadze, G. 2001, astro-ph/0105068. Ferreira, 
P.G. and Joyce, M. 1997, Phys. Rev. Lett. 79, 4740.

Frieman, J., Hill, C.T., Stebbins, A. and Waga, I. 1995, Phys. Rev. Lett. 75, 2077.

Huey G. and Lidsey, J. 2001 Phys. Lett. B 514, 217.

Perlmutter, S.J. et al. 1999, $A p J$ 517, 565.

Ratra, B. and Peebles, P.J.E. 1988, Phys. Rev. D 37, 3406.

Riess, A. et al. 1998, Astronomical J. 116, 1009.

Sahni, V. 2002, Class.Quant.Grav. 193435 [astro-ph/0202076].

Sahni, V. and Habib, S. 1998, Phys. Rev. Lett. 81, 1766.

Sahni, V., Saini, T.D., Starobinsky, A.A. and Alam, U. 2002, astro-ph/0201498.

Sahni, V., Sami, M. and Souradeep, T. 2002, Phys. Rev. D 65023518.

Sahni, V. and Shtanov, Yu. astro-ph/0202346.

Sahni, V. and Starobinsky, A.A. 2000, IJMP D 9, 373.

Sahni, V. and Wang, L. 2000, Phys. Rev. D 62, 103517.

Saini, T.D., Raychaudhury, S., Sahni, V. and Starobinsky, A.A., 2000, Phys.

Rev. Lett. 85, 1162.

Ureña-López, L.A. and Matos, T. 2000, Phys. Rev. D 62, 081302.

Wang, X., Tegmark, M. and Zaldarriaga, M. 2001, astro-ph/0105091.

Weinberg, S. 1989, Rev. Mod. Phys. 61, 1. Wetterich, C. 1988, Nuclear Physics B 302, 668.

Zeldovich, Ya.B. 1968, Sov. Phys. - Uspekhi 11, 381.

Zlatev, I., Wang, L. and Steinhardt, P.J. 1999, Phys. Rev. Lett. 82, 896. 